\title{
Lodo de esgoto e fertilizante mineral sobre parâmetros do solo e de plantas de trigo
}

\author{
Wagner A. Chueiri ${ }^{1}$, Beatriz M. Serrat ${ }^{2}$, Jonathan Biele ${ }^{3} \&$ Nerilde Favaretto $^{2}$
}

\begin{abstract}
RESUMO
Para avaliar o lodo de esgoto alcalinizado (LEA), como complemento da adubação mineral instalou-se, em junho de 1997, um experimento em vasos com a cultura do trigo, cultivar BR 35, em casa de vegetação no Setor de Ciências Agrárias da Universidade Federal do Paraná, em Curitiba, PR, no qual se empregou o fatorial $5 \times 5$, combinando 0,25 , 50,75 e $100 \%$ da adubação mineral recomendada para a cultura, com 0, 2,5, 5,0, 7,5 e 10,0 Mg ha ${ }^{-1}$ do LEA em base seca. O LEA, aplicado ao solo vinte dias antes da semeadura e adubação mineral do trigo, elevou o pH acima da neutralidade. A saturação por bases, capacidade de troca de cátions e teores de P, K, Ca, Cu e Zn aumentaram, enquanto o Mn e $\mathrm{H}+\mathrm{Al}$ diminuíram. O LEA afetou negativamente os parâmetros morfológicos e a matéria seca das plantas e ainda elevou os teores de $\mathrm{K}, \mathrm{N}, \mathrm{Mg}$, Cu e Zn da parte aérea do trigo, enquanto o fertilizante os reduziu, indicando efeito de concentração e diluição, respectivamente. A elevação do pH do solo reduziu a disponibilidade de $\mathrm{Mn}$ para o trigo, danificando a cultura.
\end{abstract}

Palavras-chave: biossólido alcalino, fertilidade do solo, micronutrientes, nutrição de plantas, Triticum durum L.

\section{Sewage sludge and mineral fertilizer effect on soil and wheat crop parameters}

\begin{abstract}
In order to evaluate the effect of an alkaline sewage sludge (ASS) as a supplement for soil chemical fertilization, an experiment with wheat crop was carried out in greenhouse. The experimental design was a $5 \times 5$, combining 5 rates of chemical fertilizers $(0,25,50,75$ and $100 \%$ of rates recommended at planting) with 5 rates of dried ASS $(0,2.5,5.0$, 7.5 and $10.0 \mathrm{Mg} \mathrm{ha}^{-1}$ ). The ASS, applied 20 days before seeding, raised the soil $\mathrm{pH}$ above neutral. Base saturation, cation exchange capacity and the contents of $\mathrm{P}, \mathrm{K}, \mathrm{Ca}, \mathrm{Cu}$ and $\mathrm{Zn}$ were increased by the ASS application, whereas the contents of $\mathrm{Mn}$ and $\mathrm{H}+\mathrm{Al}$ were decreased. The ASS negatively affected the morphological parameters and the dry-matter yield and increased the contents of $\mathrm{K}, \mathrm{N}, \mathrm{Mg}, \mathrm{Cu}$ and $\mathrm{Zn}$ in wheat plants, whereas the fertilizer application provided opposite results, suggesting the effect of concentration and dilution for the ASS and fertilizer, respectively. The increased soil $\mathrm{pH}$ reduced the $\mathrm{Mn}$ availability to wheat plants, resulting in crop damage.
\end{abstract}

Key words: alkaline biosolid, soil fertility, $\mathrm{pH}$, micronutrients, yield wheat

\footnotetext{
${ }^{1}$ Engenheiro Agrônomo, Mestre em Ciência do Solo. Rua Tibagi, 294, sala 808, CEP 80060-110, Curitiba, PR. Fone: (41) 3223-0584. E-mail: wagner.chueiri@bunge.com 2 DSEA/UFPR, Rua dos Funcionários 1540, Cabral, CEP 80035-050, Curitiba, PR. Fone: (41) 3350-5638; 3350-5609. E-mail: nfavaretto@ufpr.br; bmserrat@ufpr.br 3 DE/UFPR. Ed. da Administração, 3.andar, CP 19081, CEP 81531-990, Jardim das Américas, Curitiba, PR. Fone: (41) 3364-3006. E-mail: m4273@terra.com.br
} 


\section{INTRODUÇÃO}

O último censo demográfico mostra que 81,2\% da população brasileira vivem em áreas urbanas (IBGE, 2001), indicando a necessidade do aumento da eficiência das áreas agrícolas na produção de alimentos. Esta população urbana produz constantemente grandes quantidades de dejetos que, em escala variada, são coletados pelo sistema de esgoto sanitário das cidades. O esgoto urbano, quando submetido a tratamento adequado, gera o chamado "lodo de esgoto" ou biossólido, o qual é produzido em 66 municípios do Estado

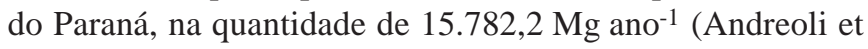
al., 1998), e é passível de disposição agrícola depois de submetido a desinfecção, através de processos específicos. Em alguns trabalhos se tem avaliado essa alternativa de disposição. Bettiol et al. (1983), verificaram que o lodo de esgoto digerido $(\mathrm{pH}=6,03)$ em dose equivalente a $9,0 \mathrm{Mg} \mathrm{ha}^{-1} \mathrm{em}$ base seca, pode ser utilizado como fonte de nutrientes para o milho (Zea mays) e arroz (Orizae sativa), pois não existe diferença estatisticamente significativa se comparado com os tratamentos que receberam fertilização mineral recomendada. Berton et al. (1989), trabalhando com lodo anaerobicamente digerido em doses equivalentes a 0 , 40 e $80 \mathrm{Mg} \mathrm{ha}^{-1}$ em base seca, concluíram aumento significativo na produção de matéria seca e na absorção de N, P, Ca, Mg e Zn pelas plantas de milho em todos os solos, nas duas doses. O lodo ainda proporcionou aumento do $\mathrm{pH}$, do teor de matéria orgânica, dos teores de Ca, $\mathrm{Mg}$ e $\mathrm{K}$ trocáveis e do teor de $\mathrm{P}$ extraível em $\mathrm{H}_{2} \mathrm{SO}_{4} 0,05 \mathrm{~N}$, além da redução ou eliminação do Al trocável, nos cinco solos estudados, após 15 dias da sua aplicação. Rawajfih \& Gharaibeh (1990), cultivando trigo (Triticum durum L.) durante 60 dias em vasos preenchidos com mistura de lodo seco ( $\mathrm{pH}$ em $\mathrm{H}_{2} \mathrm{O}=7,0$ ) e solo calcário (pH em $\mathrm{H}_{2} \mathrm{O}=8,0$ ) nas proporções de 1:8, 1:6, 1:4, 1:2, 1:1, $2: 1$ e $4: 1$, constataram que a mistura $1: 8$ aumentou significativamente o crescimento das plantas de trigo em relação à testemunha com ausência de lodo. A proporção 1:6 não teve efeito significativo enquanto as demais misturas apresentaram efeito supressivo sobre o desenvolvimento do trigo. Em trabalho com lodo de esgoto de várias origens, Sloan \& Basta (1995) usaram, em experimento de incubação, três solos fortemente ácidos, com os seguintes tratamentos: calcário, lodo alcalino estabilizado, lodo anaeróbio não alcalinizado e $\mathrm{N}$-viro\#, verificando que, em geral, o pH dos solos aumentou linearmente com as doses dos produtos aplicados, para os três solos estudados. Os tratamentos promoveram redução do teor de Mn em solução e também do Al solúvel, com exceção do lodo anaeróbio não alcalinizado, cuja redução atingiu níveis inferiores ao limite de detecção $\left(<0,4 \mathrm{~mol} \mathrm{~L}^{-1}\right)$. A especiação mostrou que a atividade do $\mathrm{Al}^{3+}$ foi reduzida para níveis não tóxicos $\left(<10 \mathrm{mmol} \mathrm{L}^{-1}\right)$. Nas condições do Estado do Paraná, Favaretto et al. (1997) conduziram experimento em campo, com a cultura do milho, utilizando 32, 64 e $96 \mathrm{Mg} \mathrm{ha}^{-1}$ de lodo úmido alcalinizado, produzido na ETE-Belém, comparando-o com a adubação mineral recomendada, cujos resultados indicaram não haver diferenças de produtividade entre a adubação mineral e qualquer dose de lodo e, ainda, que o lodo reduziu a acidez potencial do solo e aumentou os teores de $\mathrm{P}, \mathrm{Ca}+\mathrm{Mg}$ e $\mathrm{V} \%$, mas não afetou significativamente o $\mathrm{pH}$ nem teores de $\mathrm{K}$, C e CTC a pH 7,0 do solo.

Com o presente trabalho, objetivou-se testar a aplicação do LEA em complemento à adubação mineral de plantas de trigo (Triticum aestivum), em solo previamente corrigido, avaliando-se os parâmetros químicos do solo e nutricionais da cultura. Espera-se que, devido à sua composição química, o LEA possa aportar nutrientes ao solo e substituir parte do fertilizante mineral, sendo aplicado na pré-semeadura do trigo.

\section{MATERIAL E MÉTODOS}

O experimento foi conduzido em casa de vegetação do Setor de Ciências Agrárias da UFPR, em Curitiba, PR. O solo foi coletado na camada de 0 a $20 \mathrm{~cm}$ (IAPAR, 1996), no campo experimental da Cooperativa Agropecuária Batavo Ltda., situado no km 121 da Rodovia PR 151, em Ponta Grossa, PR (2520’ S; 50²0’ W), classificado como Latossolo Vermelho distrófico (LVd), (EMBRAPA, 1999). Realizou-se a caracterização química do solo (Tabela 1) de acordo com metodologia proposta por Pavan et al. (1992) para análise de rotina, enquanto a determinação dos micronutrientes se baseou na metodologia de Lindsay \& Norvell (1978), empregando-se o extrator DTPA-TEA.

O solo apresentou densidade aparente de $1,54 \mathrm{~kg} \mathrm{dm}^{-3}$, a qual foi determinada pelo método do anel volumétrico e granulometria composta por $780 \mathrm{~g} \mathrm{~kg}^{-1}$ de areia, $85 \mathrm{~g} \mathrm{~kg}^{-1}$ de silte e $135 \mathrm{~g} \mathrm{~kg}^{-1}$ de argila, determinada pelo método do densímetro (EMBRAPA, 1997).

O lodo de esgoto alcalinizado (LEA) foi produzido em sistema aeróbio, pela Companhia de Saneamento do Paraná - SANEPAR, em sua Estação de Tratamento de Esgotos Belém (ETE-Belém), situada em Curitiba, PR. A análise química do lodo foi realizada pelo Instituto de Tecnologia do Paraná - TECPAR (Tabela 2), cujos elementos, Cd, Cr, $\mathrm{Zn}, \mathrm{Cu}, \mathrm{Ni}, \mathrm{Pb}, \mathrm{Ca}$ e $\mathrm{Mg}$, foram determinados de acordo com CETESB (1977) e o Hg por metodologia desenvolvida pelo TECPAR (Instituto de Tecnologia do Paraná). Definiram-se os teores de $\mathrm{N}_{(\text {total) }}, \mathrm{P}_{\text {(total) }}$, $\mathrm{P}_{(\mathrm{CNA})}, \mathrm{K}_{\text {(água) }}$, $\mathrm{pH}$, matéria orgâ-

Tabela 1. Análise química do solo em amostras de campo na profundidade de 0 a $20 \mathrm{~cm}$

\begin{tabular}{|c|c|c|c|c|c|c|c|c|c|c|c|c|c|}
\hline pH & $\left.A\right|^{3}$ & $\mathrm{H}+\mathrm{Al}$ & $\mathrm{Ca}^{2+}$ & $\mathrm{Mg}^{2+}$ & $\mathrm{K}^{+}$ & CTC & \multirow{2}{*}{$\begin{array}{c}P \\
\mathrm{mg} \mathrm{dm} \mathbf{m}^{-3}\end{array}$} & \multirow{2}{*}{$\begin{array}{c}C \\
g{d m^{-3}}^{-}\end{array}$} & \multirow{3}{*}{$\begin{array}{l}\text { V } \\
\%\end{array}$} & $\mathrm{Cu}^{2+}$ & $\mathrm{Fe}^{2+}$ & $\mathrm{Mn}^{2+}$ & $\mathrm{Zn}^{2+}$ \\
\hline $\mathrm{CaCl}_{2}$ & \multicolumn{6}{|c|}{$\mathrm{cmol}_{\mathrm{c}} \mathrm{dm}^{-3}$} & & & & \multicolumn{4}{|c|}{$\mathrm{mg} \mathrm{kg}^{-1}$} \\
\hline 6,2 & 0,0 & 3,2 & 3,2 & 2,2 & 0,25 & 8,9 & 44,5 & 10,6 & & 0,51 & 15,7 & 2,9 & 1,6 \\
\hline
\end{tabular}

\footnotetext{
\# Processo patenteado em que se emprega pó de forno de cimento alcalino, para estabilização e alcalinização do lodo de esgoto
} 
nica total e cinzas, segundo LANARV (1988); somente o teor de Mn não o foi, porém o teor médio para o lodo de esgoto do Paraná, adotado para o presente trabalho, é de $225 \mathrm{mg} \mathrm{kg}^{-1}$ (Pigozzo et al., 1998).

Tabela 2. Composição química (em base seca) do lodo de esgoto alcalinizado da ETE-Belém analisado pelo Instituto de Tecnologia do Paraná - TECPAR

\begin{tabular}{ll}
\hline C orgânico total: $203 \mathrm{~g} \mathrm{~kg}^{-1}$ & Ca: $126 \mathrm{~g} \mathrm{~kg}^{-1}$ \\
M0 total: $365 \mathrm{~g} \mathrm{~kg}^{-1}$ & $\mathrm{Mg}: 73 \mathrm{~g} \mathrm{~kg}^{-1}$ \\
Umidade $\left(110 \pm 5^{\circ} \mathrm{C}\right): 22,7 \mathrm{~g} \mathrm{~kg}^{-1}$ & $\mathrm{Cu}: 120 \mathrm{mg} \mathrm{kg}^{-1}$ \\
Cinzas $\left(550 \pm 5^{\circ} \mathrm{C}\right): 613 \mathrm{~g} \mathrm{~kg}^{-1}$ & $\mathrm{Zn}: 549 \mathrm{mg} \mathrm{kg}^{-1}$ \\
Relação $\mathrm{C} / \mathrm{N}(\mathrm{C}$ total$/ \mathrm{N}$ total): $7 / 1$ & $\mathrm{Cd}: 8,5 \mathrm{mg} \mathrm{kg}^{-1}$ \\
$\mathrm{pH}: 11,6$ & $\mathrm{Cr}: 40 \mathrm{mg} \mathrm{kg}^{-1}$ \\
$\mathrm{~N}$ total: $30,6 \mathrm{~g} \mathrm{~kg}^{-1}$ & $\mathrm{~Pb}: 43 \mathrm{mg} \mathrm{kg}^{-1}$ \\
$\mathrm{P}_{2} \mathrm{O}_{5}$ total: $20,3 \mathrm{~g} \mathrm{~kg}^{-1}$ & $\mathrm{Hg}: 2,1 \mathrm{mg} \mathrm{kg}^{-1}$ \\
$\mathrm{P}_{2} \mathrm{O}_{5}$ (solúvel em CNA): $14,1 \mathrm{~g} \mathrm{~kg}^{-1}$ & $\mathrm{Ni}: 50 \mathrm{mg} \mathrm{kg}^{-1}$ \\
$\mathrm{~K}_{2} \mathrm{O}$ (solúvel em H $\left.\mathrm{H}_{2} \mathrm{O}\right): 2,2 \mathrm{~g} \mathrm{~kg}^{-1}$ & \\
\hline
\end{tabular}

O experimento foi conduzido em vasos com capacidade de 9,3 L e os tratamentos equivaleram às doses de 0, 2,5, 5,0, 7,5 e 10,0 $\mathrm{Mg} \mathrm{ha}^{-1}$ de LEA em base seca. As doses de fertilizante mineral foram 0, 25, 50, 75 e $100 \%$ da dose de $\mathrm{N}$, $\mathrm{P}_{2} \mathrm{O}_{5}$ e $\mathrm{K}_{2} \mathrm{O}$ (15, 30 e $45 \mathrm{~kg} \mathrm{ha}^{-1}$, respectivamente) recomendada para a cultura do trigo (OCEPAR, 1995) com base nos resultados da análise química do solo.

A mistura solo-LEA foi realizada em homogeneizador elétrico, adicionando-se o LEA diluído gradualmente, até atingir a dose correspondente a cada tratamento. Para as adições gradativas utilizaram-se $0,811,1,623,2,435$ e 3,247 g de LEA em base seca para cada kg de solo seco correspondendo, respectivamente, às doses de 2,5, 5,0, 7,5 e 10,0 $\mathrm{Mg} \mathrm{ha}^{-1}$; após o preenchimento, os vasos foram mantidos em umidade próxima à capacidade de campo, durante 20 dias, até o plantio do trigo. A aplicação do fertilizante (mistura microgranulada da fórmula 4-14-8, complementada com $\mathrm{KCl}$ e $\left.\left(\mathrm{NH}_{4}\right)_{2} \mathrm{SO}_{4}\right)$ foi realizado no sulco aberto ao longo do diâmetro do vaso, a $8 \mathrm{~cm}$ de profundidade e, ao longo do sulco já coberto, 10 orifícios eqüidistantes, com profundidade de $3 \mathrm{~cm}$, foram feitos, nos quais se depositaram as sementes de trigo, cultivar BR 35, cujo ciclo e porte são intermediários, já tratadas com fungicida triadimenol a 40 g para cada $100 \mathrm{~kg}$ de sementes; apenas as três plantas mais vigorosas foram conduzidas.

O solo misturado ao LEA foi amostrado antes da aplicação do fertilizante em seis pontos aleatórios, ao longo de toda a profundidade do vaso. As análises químicas de rotina e dos micronutrientes $\mathrm{Cu}, \mathrm{Fe}, \mathrm{Mn}$ e $\mathrm{Zn}$, basearam-se nas metodologias propostas por Pavan et al. (1992) e Lindsay \& Norvell (1978), respectivamente, enquanto as avaliações das plantas foram feitas no estádio fenológico 10.1 da escala de Feeks \& Large (Large, 1954), determinando-se o diâmetro do colmo, estatura e matéria seca (peso constante a $65^{\circ} \mathrm{C}$ ). Os teores de $\mathrm{P}, \mathrm{Ca}, \mathrm{Mg}, \mathrm{K}, \mathrm{Cu}, \mathrm{Fe}, \mathrm{Mn}$ e $\mathrm{Zn}$ no tecido vegetal (parte aérea) foram analisados através da metodologia descrita por Perkin \& Elmer (1973). Realizouse a determinação do $\mathrm{N}$ através do método do semi-micro
Kjeldahl (Sarruge \& Haag, 1974).

Os dados de solo foram submetidos à análise de regressão para se testar a significância dos modelos linear simples $(\mathrm{y}=\mathrm{a}+\mathrm{bx})$ e quadrático $\left(\mathrm{y}=\mathrm{a}+\mathrm{bx}+\mathrm{cx}^{2}\right)$. Os modelos foram plotados com seus respectivos intervalos de predição (Charnet et al., 1999). Para as avaliações de planta foram feitas regressões usando-se as duas variáveis independentes ou co-variadas (lodo e fertilizante mineral) e uma variável dependente, para produzir a superfície de resposta, dada pela equação de resposta simples, ou seja, univariada (Charnet et al., 1999). O nível de significância usado nas análises foi de 5\%. Precedendo-se as análises, as co-variadas foram submetidas a transformação canônica (Montgomery, 1997) para uniformizar seus respectivos valores nos eixos dos gráficos de superfície de resposta. Com vistas a executar a transformação, tomou-se o valor central de cada escala, que foi o subtraendo de qualquer valor da mesma e, ao mesmo tempo o divisor do resultado da subtração, como mostram as seguintes equações:

$$
\text { lodo }=(\text { Lodo }-5) / 5 \text { e fert }=(\text { Fertilizante }-50) / 50
$$

Para ambas as co-variadas, o resultado final foi: -1,0; -0,5; $0 ; 0,5$ e 1 . Devido a essas transformações todas as equações que acompanham os modelos deverão ter os valores de $\mathrm{x}$ ou y (no caso das superfícies de resposta) substituídos pelos valores transformados (-1 a 1$)$ caso se queira calcular o valor de uma variável dependente qualquer. A avaliação da correlação entre duas variáveis dependentes sob efeito simultâneo do fertilizante e lodo, foi feita empregando-se a análise de variância de resposta múltipla - MANOVA (Johnson \& Wichern, 1998). O teste de significância da MANOVA foi o Lambda de Wilks (Montgomery, 1997).

\section{RESULTADOS E DISCUSSÃO}

A avaliação do solo aos 20 d após a aplicação do LEA, mostrou elevação do $\mathrm{pH}$, da saturação por bases (V\%) e dos teores de $\mathrm{P}$ e $\mathrm{Ca}$, enquanto o $\mathrm{Mn}$ e $\mathrm{H}+\mathrm{Al}$ tiveram seus teores reduzidos (Figura 1 ).

A CTC e os teores de $\mathrm{K}$, Cu e Zn, também aumentaram em função da dose de LEA aplicada. Devido aos baixos coeficientes de determinação $\left(\mathrm{r}^{2}\right)$ do modelo matemático, esses e seus respectivos níveis de significância estão apresentados na Tabela 3.

A elevação do $\mathrm{pH}$ do solo, em função da aplicação do LEA, também foi observada por Berton et al. (1989), Brown \& Brush (1992), Sloan \& Basta (1995). Em virtude do LEA

Tabela 3. Coeficiente de determinação $\left(\mathrm{r}^{2}\right)$ para a CTC e os teores de K, Cu e Zn no solo, em função das doses de lodo de esgoto alcalinizado, após $20 \mathrm{~d}$ da aplicação

\begin{tabular}{cccc}
\hline Variável & $\mathrm{r}^{2}$ & Variável & $\mathrm{r}^{2}$ \\
CTC & $0,142^{\star * *}$ & $\mathrm{Cu}$ & $0,095^{\star}$ \\
$\mathrm{K}$ & $0,088^{* *}$ & $\mathrm{Zn}$ & $0,199 * *$ \\
\hline
\end{tabular}

* Significativo a $5 \%,{ }^{* \star}$ a $1 \%$ e ${ }^{* \star *}$ a $0,1 \%$ no teste $F$ 
A.

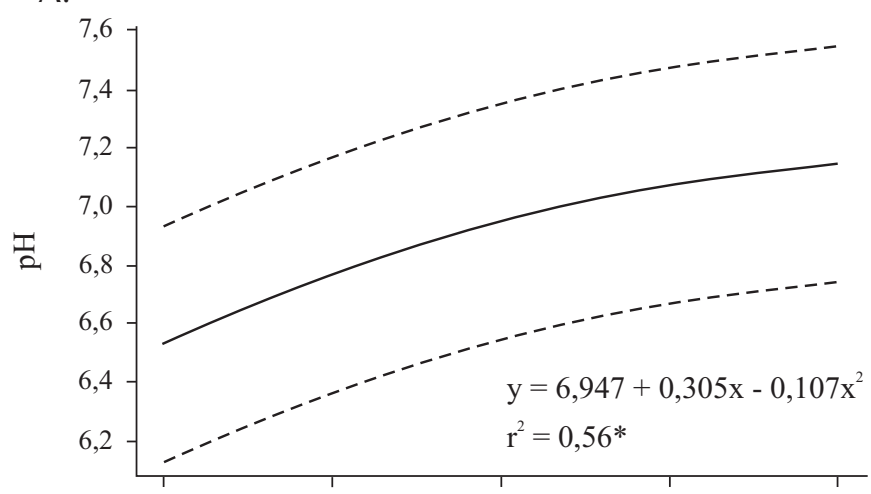

B.

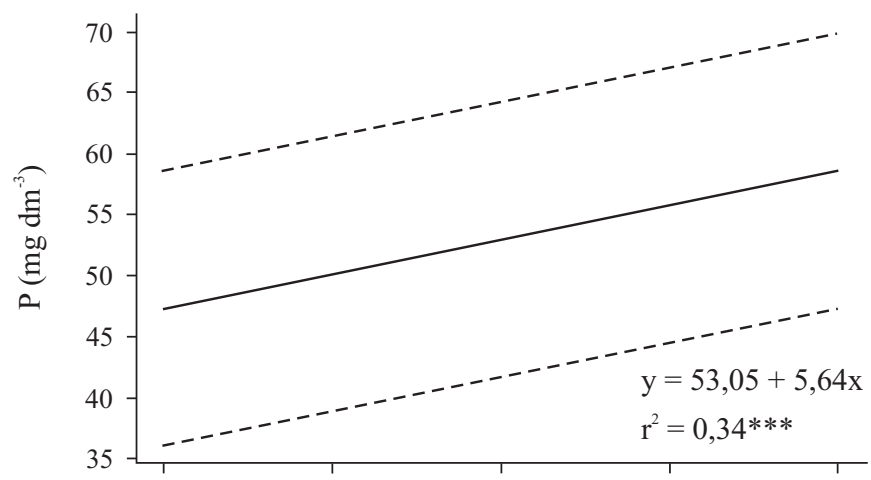

C.

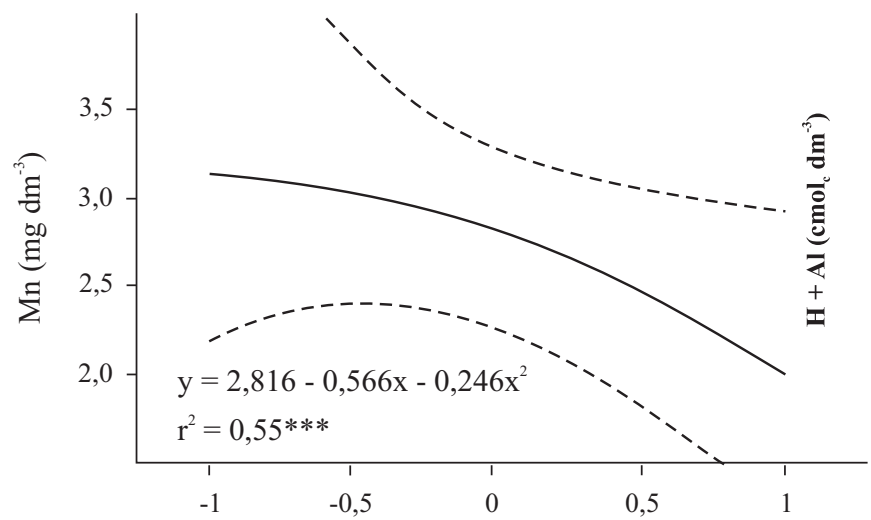

D.

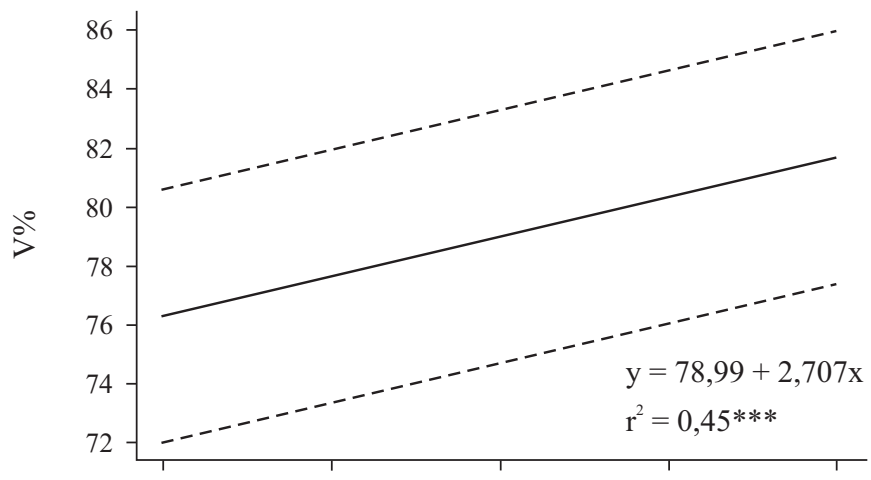

E.

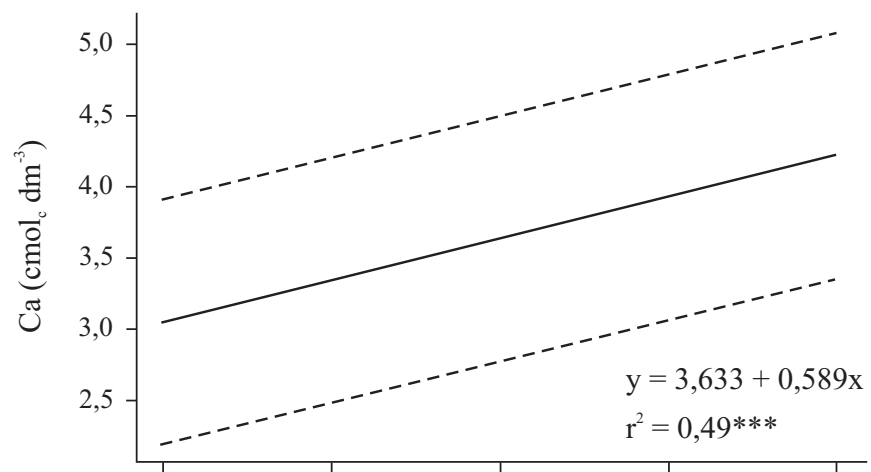

F.

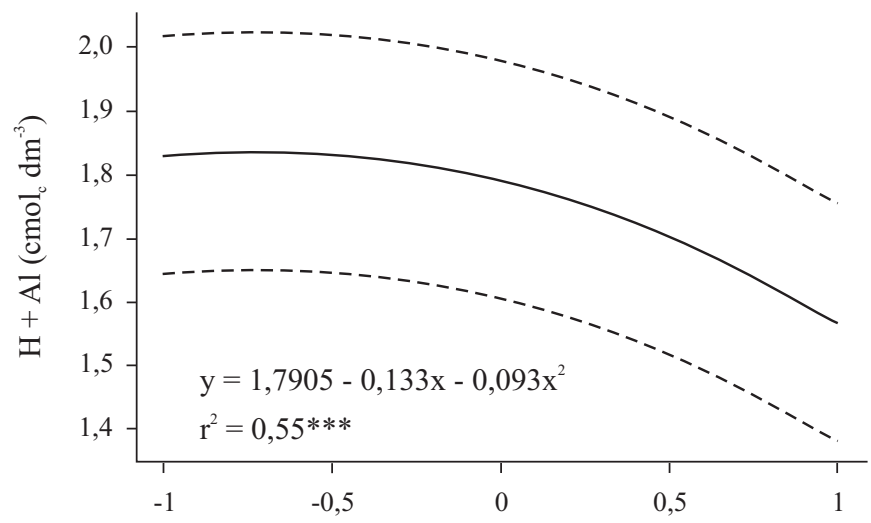

Lodo

Figura 1. Equações de regressão para o $\mathrm{pH}(\mathrm{A}), \mathrm{P}(\mathrm{B}), \mathrm{Mn}(\mathrm{C}), \mathrm{V} \%$ (D), Ca (E) e H+Al (F) do solo, em função das doses de lodo de esgoto alcalinizado em valores transformados, após 20 dias da aplicação e respectivos intervalos de predição (linha pontilhada). Os valores do eixo $(-1$ a 1$)$ equivalem às doses transformadas de lodo de esgoto de 0 a $10 \mathrm{Mg} \mathrm{ha}^{-1}$, respectivamente

ser um material altamente alcalino e de reatividade elevada, o aumento do pH e conseqüente redução da acidez potencial $(\mathrm{H}+\mathrm{Al})$, ocorreram em curto intervalo de tempo mesmo se tratando de um solo com baixa acidez. Meyer (1998) verificou que o efeito corretivo do LEA é praticamente imediato atingindo, com freqüência, os valores máximos de pH após um dia da aplicação. O Ca é o nutriente que se encontra em maior quantidade no LEA (Tabela 2); portanto, a elevação de seus teores no solo e o aumento da saturação por bases (V\%) foram condizentes com as doses crescentes aplicadas. A adição do LEA propiciou a elevação do teor de P no solo; todavia, considerando-se a concentração desse elemento no biossólido, conclui-se que os teores obtidos na análise de solo ficaram aquém daqueles potencialmente possíveis; este fato pode estar relacionado aos elevados valores de $\mathrm{pH}$ do solo que, além de alterar a solubilidade dos compostos com P, interferem na eficiência do extrator Mehlich1 (Novais \& Smyth, 1999). Os teores de Mg, C e Fe não foram afetados pelo LEA, após 20 dias da aplicação. 
O C orgânico total (20,3\%), presente no LEA, não aumentou significativamente o teor de C no solo, semelhante ao resultado obtido por Favaretto et al. (1997); no entanto, Melo et al. (1994) obtiveram aumentos não apenas do C orgânico, mas também da CTC do solo, aos 77 dias após a aplicação, de 32,0 Mg ha-1 de lodo de esgoto em base seca, dose esta três vezes superior à máxima dose empregada no presente trabalho. Mesmo não ocorrendo aumento significativo no teor de C orgânico no solo, o lodo promoveu o aumento significativo da CTC a pH 7,0, podendo estar relacionado à característica dos ácidos húmicos que compõem sua fração orgânica.

O Mn foi o único micronutrientre cujo teor foi significativamente reduzido com a aplicação do LEA (Figura 1C); este comportamento está relacionado à grande sensibilidade desse elemento à elevação do pH, formando compostos muito pouco solúveis quando os valores de $\mathrm{pH}$ em água se aproximam de 6,4 (Lindsay \& Norvell, 1978). Embora o pH inicial do solo já apresentasse valor elevado (6,2 em $\mathrm{CaCl}_{2}$ $0,01 \mathrm{M}$ ), somente nas maiores doses de LEA é que se observaram baixos níveis de Mn extraível (DTPA-TEA) no solo.

O LEA afetou negativamente algumas características morfológicas do trigo e a produção de matéria seca, enquanto o fertilizante exerceu efeito benéfico sobre os mesmos parâmetros (Figura 2) indicando que, apesar de adicionar nutrientes ao solo, o LEA deverá ter sua aplicação controlada; esta observação corrobora a decisão atual da Companhia de Saneamento do Paraná - SANEPAR, em definir a dose de LEA em função do poder corretivo (PROSAB, 1999).

No tecido vegetal, os teores de $\mathrm{P}$, Ca e Fe não sofreram alterações em virtude da aplicação de fertilizante e/ou LEA; já os teores de N (Figura 3A), K (Figura 3B), Mg (Figura 3C) e $\mathrm{Cu}$ (Figura 3D) foram significativamente reduzidos pela aplicação do fertilizante e aumentados pela aplicação do LEA. Os teores de Mn (Figura 3E) e Zn (Figura 3F) foram alterados apenas pela aplicação do LEA, sendo que o primeiro elemento teve seu teor aumentado e o segundo diminuído em função das doses crescentes do biossólido. Esses resultados indicam que o fertilizante causou efeito de diluição sobre os elementos $\mathrm{N}, \mathrm{K}, \mathrm{Mg}$ e $\mathrm{Cu}$, por favorecer a produção de matéria seca em maior velocidade que a da absorção desses nutrientes, enquanto o LEA exerceu efeito de concentração sobre esses elementos, inclusive o $\mathrm{Zn}$, devido à redução da matéria seca provocada, dentre outros motivos, pela limitação na absorção do Mn; este fenômeno é conhecido como efeito de Steenjberg (Malavolta et al., 1997).

O Mn foi o único elemento que teve redução em seu teor no tecido vegetal, acompanhando a redução na produção de matéria seca, pela aplicação de doses crescentes de LEA; seus teores ficaram aquém daqueles considerados adequados para a cultura do trigo (Malavolta et al., 1997), caracterizando sua deficiência no tecido vegetal e indicando ser este o fator limitante no desenvolvimento das plantas.

Além da elevação do $\mathrm{pH}$, outra possível causa da deficiência de Mn nos tecidos da planta foi o excesso de Ca na solução do solo (Lopes, 1999), provocada pela adição de LEA. As plantas que também receberam fertilizantes foram menos afetadas, podendo ter tido melhor estabelecimento inicial, inclusive do sistema radicular, melhorando o acesso ao
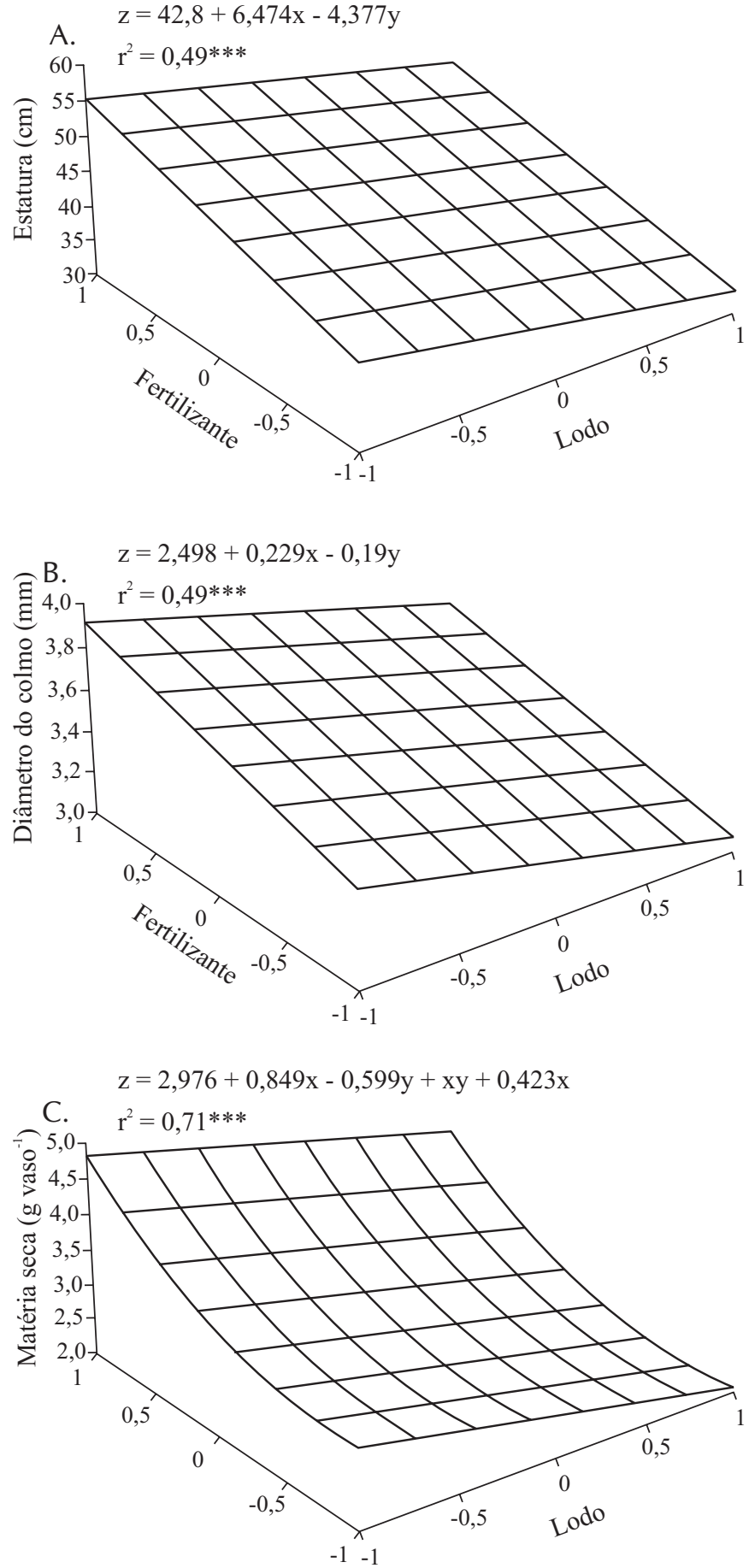

Figura 2. Equações de regressão para estatura (A), diâmetro do colmo (B) e matéria seca $(C)$ do trigo, em função das doses de lodo de esgoto alcalinizado e fertilizante mineral em valores transformados, onde: $z$ - estatura ou diâmetro do colmo ou matéria seca; $x$ - fertilizante mineral; y - lodo de esgoto em valores transformados. Os valores do eixo x e y transformados, ou seja, de -1 a 1, são equivalente a 0 a $100 \%$ da dose do fertilizante mineral, e 0 a $10 \mathrm{Mg} \mathrm{ha}^{-1}$ de lodo de esgoto, respectivamente

Mn disponível. Fato relevante é que, na adubação mineral, empregaram-se duas matérias-primas que geram reação ácida nas imediações do local de aplicação - o superfosfato simples, usado na fórmula 04-14-08 (Brady, 1989), e o sulfato de amônio, favorecendo a disponibilidade do Mn que se encontrava em formas menos solúveis, o que justifica o efeito recuperador do fertilizante sobre as plantas de trigo. 
A.

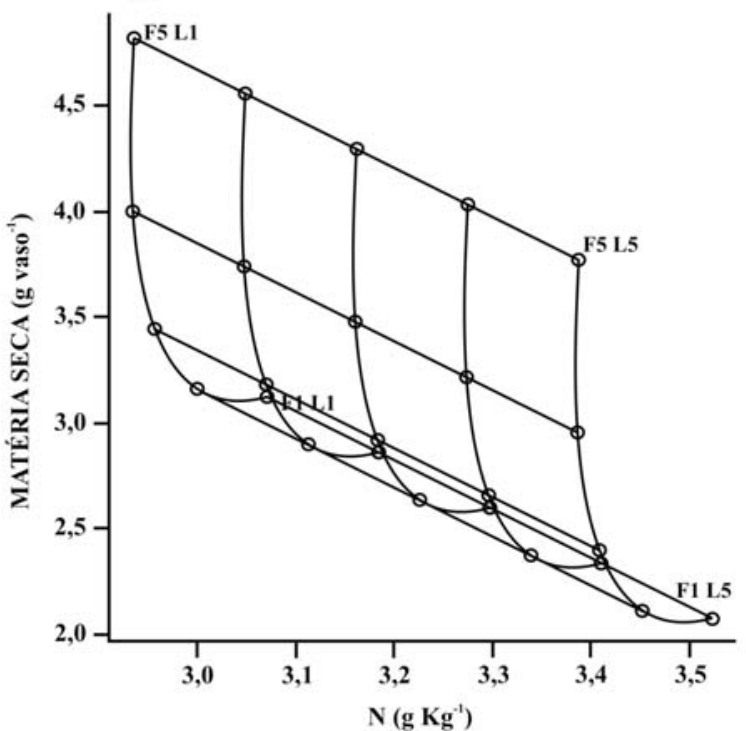

B.

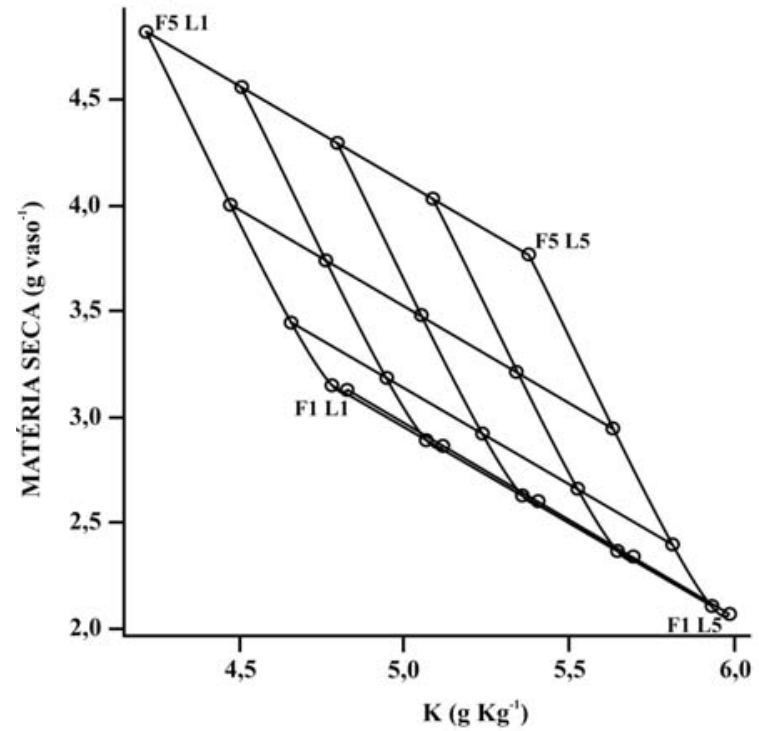

C.

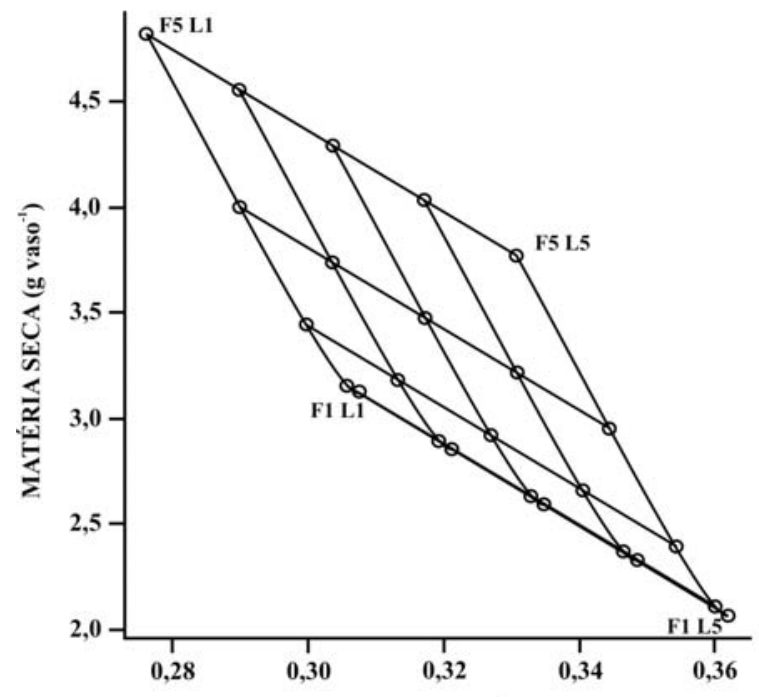

D.

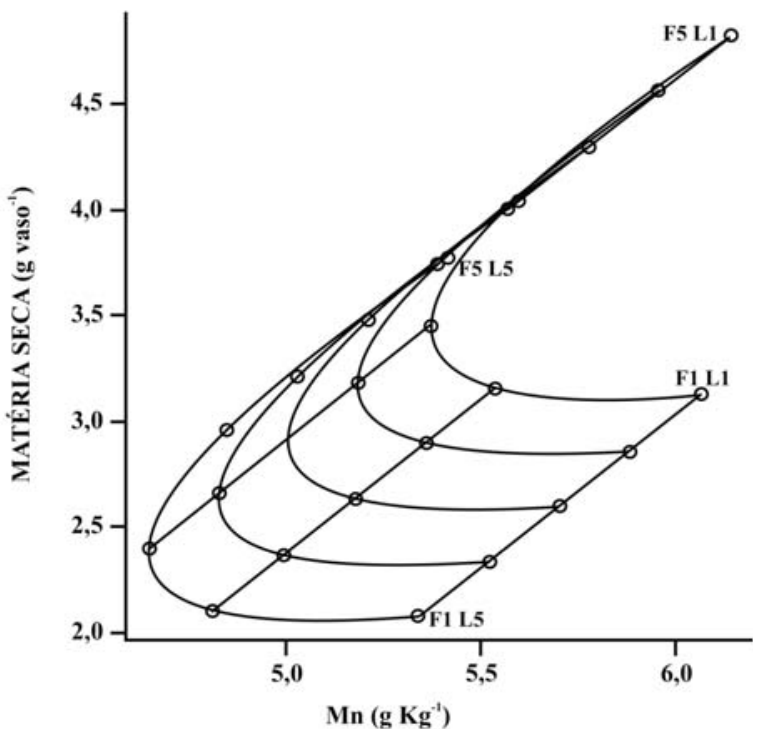

E.

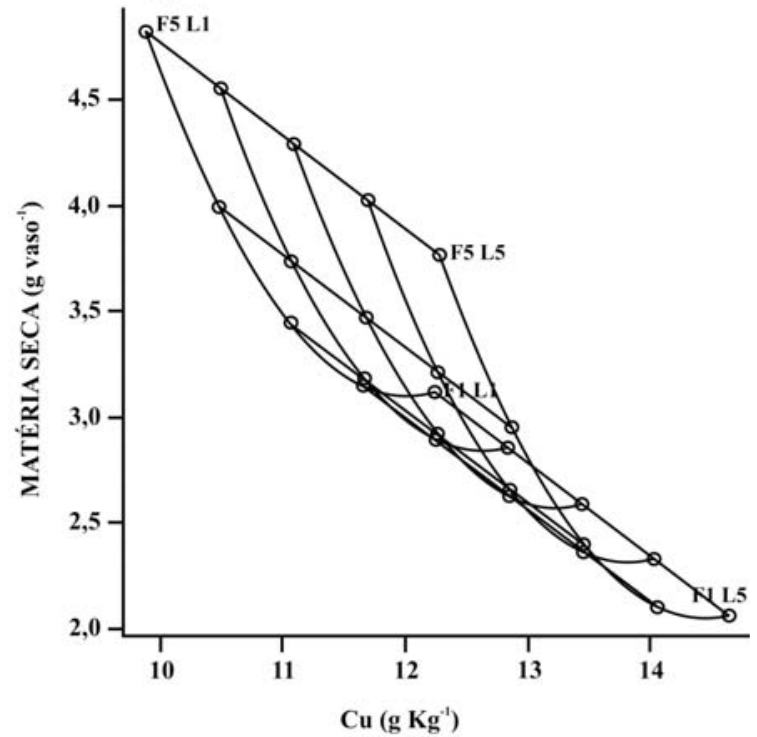

F.

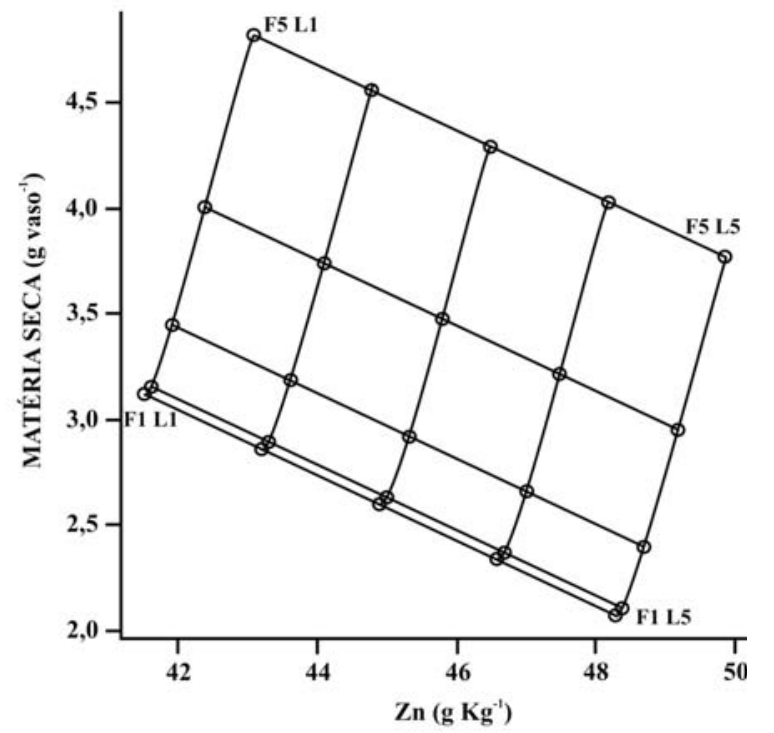

Figura 3. Curvas de resposta referente ao efeito do lodo de esgoto alcalinizado e do fertilizante mineral sobre a produção de matéria seca e teores de $\mathrm{N}$ (A), K (B), Mg (C), Mn (D), Cu (E) e Zn (F) nas plantas de trigo, onde: F1, F2, F3, F4 e F5 - dose de fertilizante mineral correspondente a 0, 25, 50, 75 e $100 \%$ da recomendação para o trigo, respectivamente, e L1, L2, L3, L4 e L5 - dose de lodo referente a $0,2,5,5,0,7,5$ e $^{2} \mathrm{Mg}_{\text {ha }}{ }^{-1}$, respectivamente 


\section{CONCLUSÕES}

1. O lodo de esgoto alcalinizado $(\mathrm{pH}=11,6)$ após 20 dias da sua aplicação ao solo, elevou o pH acima da neutralidade, aumentou a saturação por bases, a capacidade de troca de cátions e os teores de $\mathrm{P}, \mathrm{K}, \mathrm{Ca}$, $\mathrm{Cu}$ e $\mathrm{Zn}$, diminui os teores de $\mathrm{Mn}$ e $\mathrm{H}+\mathrm{Al}$ mas não afetou o carbono do solo.

2. Os parâmetros morfológicos e a matéria seca das plantas de trigo foram negativamente afetados pelo lodo de esgoto alcalinizado, enquanto o fertilizante apresentou efeito inverso.

3. Os teores de $\mathrm{K}, \mathrm{N}, \mathrm{Mg}, \mathrm{Cu}$ e $\mathrm{Zn}$ no tecido vegetal do trigo foram aumentados pelo lodo, enquanto o fertilizante mineral os reduziu, indicando o efeito de concentração e diluição, respectivamente.

4. O lodo de esgoto alcalinizado reduziu a disponibilidade de $\mathrm{Mn}$ no solo, em função da elevação do $\mathrm{pH}$, prejudicando o desenvolvimento do trigo.

\section{LITERATURA CITADA}

Andreoli, C. V.; Ferreira, A. C.; Bonnet, B.; Fernandes, F.; Lara, A. I.; Pegorini, E. S. Produção real e estimativas teóricas de lodo de esgoto no estado do Paraná. In: Seminário Sobre Gerenciamento de Biossólidos do Mercosul, 1, 1998, Curitiba. Anais... Curitiba: SANEPAR/ABES, 1998. p.231-237.

Berton, R. S.; Camargo, O. A.; Valadares, J. M. A. Absorção de nutrientes pelo milho em resposta à adição de lodo de esgoto a cinco solos paulistas. Revista Brasileira de Ciência do Solo, Campinas, v.13, p.187-192, 1989.

Bettiol, W.; Carvalho, P. C. T.; Franco, B. J. D. C. Utilização do lodo de esgoto como fertilizante. O Solo, Piracicaba, v.75, n.1, p.44-54, 1983.

Brady, N. C. Natureza e propriedades dos solos. 7.ed. Rio de Janeiro: Freitas Bastos, 1989. 898p.

Brown, J. R.; Brush, L. Lime stabilized sludge treatment acid soils relative to soil acidity minimums for sludge application. Communications of Soil Science and Plant Analysis., New York, v.23, n.11/12, p.1257-1273, 1992.

CETESB - Companhia de Tecnologia de Saneamento Ambiental. Análises físico-químicas para controle de estações de tratamento de esgotos. São Paulo: CETESB, 1977. 226p.

Charnet, R.; Freire, C. A. L.; Charnet, E. M. R.; Bonvino, H. Introdução aos modelos lineares - com aplicações. Campinas: UNICAMP, 1999. 340p.

EMBRAPA - Empresa Brasileira de Pesquisa Agropecuária. Centro Nacional de Pesquisa de Solos. Manual de métodos de análise de solos. 2.ed. Rio de Janeiro, Embrapa, 1997. 212p.

EMBRAPA - Empresa Brasileira de Pesquisa Agropecuária. Centro Nacional de Pesquisa de Solos. Sistema Brasileiro de Classificação de Solos. Rio de Janeiro, Embrapa, 1999. 412p.

Favaretto, N.; Deschamps, C.; Daros, E.; Pissaia, A. Efeito do lodo de esgoto na fertilidade do solo e no crescimento e produtividade de milho (Zea mays L.). Brazilian Archives of Biology and Technology, Curitiba, v.40, n.4, p.837-847, 1997.

IAPAR - Instituto Agronômico do Paraná. Amostragem de solo para análise química: plantio direto e convencional, culturas perenes, várzeas, pastagens e capineiras. Londrina: IAPAR,1996. 28p. Circular n. 90.

IBGE - Instituto Brasileiro de Geografia e Estatística. Resultados preliminares do censo demográfico 2000. Rio de Janeiro, IBGE, 2001. 156p.

Johnson, R. A.; Wichern, D. W. Applied multivariate statistical analysis. 4.ed. New Jersey: Prentice Hall, 1998. 816p.

LANARV - Laboratório Nacional de Referência Vegetal. Análise de corretivos, fertilizantes e inoculantes: Método oficial. Brasília: Secretaria Nacional de Defesa Agropecuária, 1988.104p.

Large, E. C. Growth stages in cereals illustration of the Feeks scales. Plant Pathology, v.4, p.22-24, 1954.

Lindsay, W. L.; Norvell, W. A. Development of a DTPA soil test for zinc, iron, manganese and cooper. Soil Science Society of America Journal. Madison, v.42, p.421-8, 1978.

Lopes, A. S. Micronutrientes: filosofias de aplicação e eficiência agronômica. São Paulo, 1999. 72p. Boletim Técnico, n.8.

Malavolta, E.; Vitti, G. C.; Oliveira, S. A. Avaliação do estado nutricional das plantas: Princípios e aplicações. Piracicaba: Potafos, 1997. 201p.

Melo, W. J.; Marques, M. O.; Santiago, G.; Chelli, R. A. Leite, S. A. S. Efeito de doses crescentes de lodo de esgoto sobre as frações da matéria orgânica e CTC de um solo Latossolo cultivado com cana-de-açúcar. Revista Brasileira de Ciência do Solo, Campinas, v.18, p.449-455, 1994.

Meyer, M. Avaliação do efeito corretivo do lodo de esgoto alcalinizado em solos ácidos. Curitiba: UFPR, 1998. 44p. Monografia Graduação

Montgomery, C. D. Desingn and analysis of experiments. 4.ed. Tempe: John Wiley \& Sons, 1997. 704p.

Novais, R. F.; Smyth, T. J. Fósforo em solo e planta em condições tropicais. Viçosa: UFV, 1999. 399p.

OCEPAR - Organização das Cooperativas do Estado do Paraná. Recomendações técnicas para a cultura do trigo no estado do Paraná. Cascavel: OCEPAR, 1995, 115p. Boletim Técnico, n.37

Pavan, M. A.; Bloch, M. de F.; Zempulski, H. da C.; Miyazawa, M.; Zocoler, D. C. Manual de análise química do solo e controle de qualidade. Londrina: IAPAR, 1992. 40p.

Perkin \& Elmer. Analytical methods for atomic absorption spectrometry agriculture. Connecticut, 1973.

Pigozzo, A. T. J.; Gobbi, M. A.; Lenzi, E.; Luchesi, E. B. Efeitos da aplicação de lodo de esgoto e resíduo petroquímico no cultivo do milho como fonte de micronutrientes no Latossolo Vermelho Escuro. In: Seminário Sobre Gerenciamento de Biossólidos do Mercosul, 1, 1998, Curitiba. Anais... Curitiba: SANEPAR/ABES, 1998. p.165-173.

PROSAB - Programa de Pesquisa e Saneamento Básico. Uso e manejo do lodo de esgoto na agricultura. Rio de Janeiro: PROSAB, 1999, 97p.

Rawajfih, Z.; Gharaibeh, S. High rates of domestic sewage sludge on calcareous soil and their effect on wheat growth using a pot experiment. Z. Pflanzenernähr. Bodenk, Weinheim, v.153, p.297-300, 1990.

Sarruge, J. R.; Haag, H. P. Análises químicas em plantas. Piracicaba: ESALQ/USP, 1974. 56p.

Sloan, J. J.; Basta, N. T. Remediation of acid soils by using alkaline biosolids. Journal of Environmental Quality, Madison, v.24, p.1097-1103, 1995. 